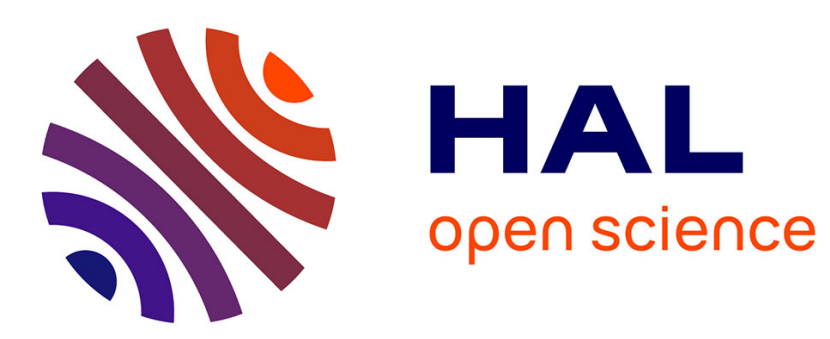

\title{
Controlling recirculation rate for minimal-time bioremediation of natural water resources
}

Alain Rapaport, Victor Riquelme

\section{To cite this version:}

Alain Rapaport, Victor Riquelme. Controlling recirculation rate for minimal-time bioremediation of natural water resources. Automatica, 2019, 106, pp.77-82. 10.1016/j.automatica.2019.04.037 . hal02077779

\section{HAL Id: hal-02077779 \\ https://hal.science/hal-02077779}

Submitted on 23 Mar 2019

HAL is a multi-disciplinary open access archive for the deposit and dissemination of scientific research documents, whether they are published or not. The documents may come from teaching and research institutions in France or abroad, or from public or private research centers.
L'archive ouverte pluridisciplinaire $\mathbf{H A L}$, est destinée au dépôt et à la diffusion de documents scientifiques de niveau recherche, publiés ou non, émanant des établissements d'enseignement et de recherche français ou étrangers, des laboratoires publics ou privés. 


\title{
Controlling recirculation rate for minimal-time bioremediation of natural water resources
}

\author{
A. Rapaport ${ }^{\mathrm{a}}$ and V. Riquelme ${ }^{\mathrm{b}}$ \\ ${ }^{a}$ MISTEA (Mathématiques, Informatique et STatistique pour l'Environnement et l'Agronomie), \\ Univ. Montpellier, Inra, Montpellier SupAgro 2, place P. Viala, 34060 Montpellier, France. \\ ${ }^{\mathrm{b}}$ Departamento de Matemática, Universidad Técnica Federico Santa María, \\ Avenida España 1680, Valparaíso, Chile
}

\begin{abstract}
We revisit the minimal time problem of in-situ decontamination of large water resources with a bioreactor, considering a recirculation flow rate in the resource as an additional control. This new problem has two manipulated inputs : the flow rate of the treatment in the bioreactor and the recirculation flow rate of the water resource between the pumping and reinjection locations. Although the velocity set of the dynamics is non convex, we show that the optimal control is reached among nonrelaxed controls. The optimal strategy consists in three sequential steps: 1. do not mix and take the flow rate of treatment that maximizes the concentration decay in the resource. 2. mix as much as possible and carry on with the flow rate that maximizes the concentration decay. 3. carry on mixing but do not treat the water. Finally, we show on numerical simulations that a significant gain in processing time can be achieved time when controlling in addition the recirculation flow rate.
\end{abstract}

Key words: Environmental engineering, minimum-time control, feedback controller, bioreactor, recirculation control.

\section{Introduction and definitions}

Water decontamination is one of the world largest issues due to scarcity of fresh water and access to drinking water. The industry of wastewater treatment has developed several technologies for the remediation of contaminated waters, but in almost any cases water decontamination is achieved in dedicated wastewater treatment plants, that consists in treating the water in bioreactors (with primary filtration and tertiary treatments depending on the contamination) and release the treated water into the environment. For natural reservoirs such as lakes, lagoons, ponds, water tables, etc., it is highly not desirable nor often even possible to empty reservoirs for treating contaminated waters in waste-water treatment plants. In many cases, it is also required to maintain the volume of the resource at a (more or less) constant volume to preserve the various forms of life (aquatic plants,

Email addresses: alain.rapaport@inra.fr (A. Rapaport), vriquelme@dim.uchile.cl (V. Riquelme), This work was achieved while the second author was at Departamento de Ingeniería Matemática and Centro de Modelamiento Matemático (UMI CNRS 2807),

Universidad de Chile, Beauchef 851, Santiago, Chile. fishes...). Refilling with fresh water while the reservoir is emptied for the treatment of contaminated water could be an alternative, but that is often not realistic or too expensive when dealing with very large volumes, especially for natural sites that are far from industrial areas. A solution consists in in-situ decontamination with recirculation thru aside bioreactor, maintaining the volume of the water resource constant. This is similar to the recirculation systems that are operated in industrial aquaculture. Indeed, fisheries make use of recirculation systems to optimize feedstock. After treatment, water with resting food is recirculated. However, in such systems, most of efforts are put on the potential recirculation of pathogens and pollutant removal is not the main consideration (cf. for instance [10]). Up to now, very few life-size decontamination experiments of large water resources have been reported in the literature, but recently the problem of modeling and piloting in real time the flow rate of the water treated in the bioreactor has been addressed in [3]. It has been shown that a minimal time feedback control of the flow rate could gain a significant processing time over any constant control, assuming either a perfectly mixed resource or distinguishing two patches in series in the water resource. In [4], it has been proved the remarkable property that the expres- 
sion of the optimal feedback control is preserved when considering one (or more) "dead" areas in the modeling of heterogeneity of the pollutant concentration in water resources. This has led to a validation of this feedback control on realistic simulations of the hydrodynamics of the resource based on the Navier-Stokes equations [1,2], justifying then the derivation of optimal control laws from simple o.d.e. models, without requiring the precise knowledge of the heterogeneity.

The optimal solution derived in [3] shows that the distribution of the pollutant concentrations between the two patches in series can have a positive or negative effect on the processing time, depending on how far the resource is from being fully decontaminated. This property has not been exploited to improve the processing time, up to our knowledge. In the present work, we analyze the benefit of mixing water between the two patches. Moreover, differently to [3], we also allow the concentration at the effluent of the bioreactor to be larger than the one of the area where it is released, making a re-contamination of this area (the effluent concentration remaining however bounded from above by the one of the other area from where the bioreactor is fed). This possibility might be considered useless at first glance but we shall show that it could be indeed beneficial. As in [3], the patches represent two distinct areas of the resource, of volumes $V_{1}$ and $V_{2}$, where water is respectively pumping out to be treated and reinjected back after been treated. Here, we consider the flow rate $Q_{2}$ of a recirculation pump between the two patches as an additional control of the primary flow rate $Q_{1}$ of the water treated by the bioreactor, as depicted on Fig. 1. The pollutant concentrations in the two patches are denoted $s_{1}$ and $s_{2}$, while $s_{r}$ and $x_{r}$ represent the pollutant and biomass concentrations in the bioreactor of volume $V_{r}$. To avoid an increase of eutrophication in the water resource, we assume a perfect separation of biomass in a settler at the output of the bioreactor so that no biomass is propagated in the resource. Remark that for values of the flow rate $Q_{1}$ that would lead the bioreactor to the washout of the biomass, one can also choose to by-pass the bioreactor to preserve its biomass for future uses.

\section{Assumptions and preliminaries}

The dynamics of the pollutant concentrations in the resource is

$$
\begin{aligned}
& \frac{d s_{1}}{d t}=\frac{Q_{1}+Q_{2}}{V_{1}}\left(s_{2}-s_{1}\right) \\
& \frac{d s_{2}}{d t}=\frac{Q_{1}}{V_{2}}\left(s_{r}-s_{2}\right)+\frac{Q_{2}}{V_{2}}\left(s_{1}-s_{2}\right)
\end{aligned}
$$

(where the volumes are assumed to be constant), and we consider the well-known chemostat model to describe

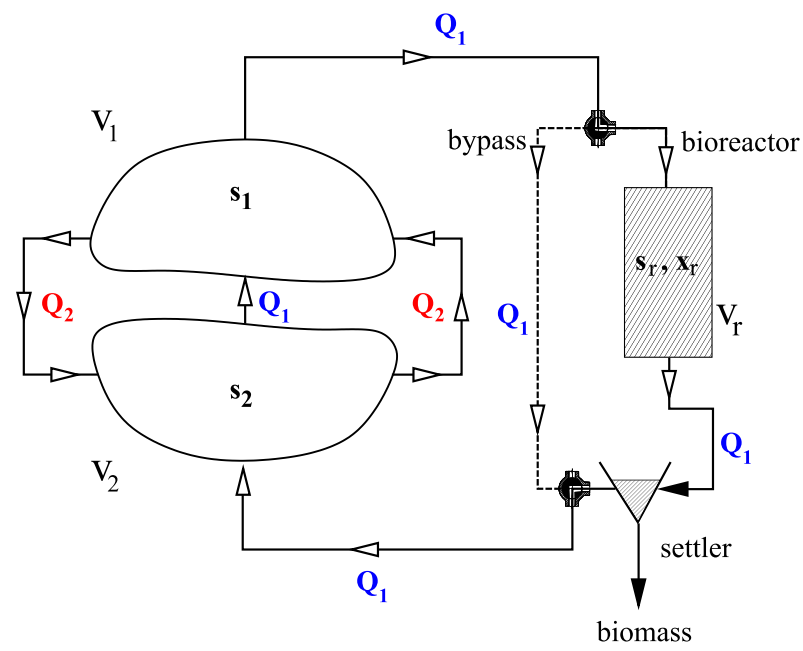

Figure 1. Interconnections scheme.

the dynamics of the bioreactor [5]:

$$
\begin{aligned}
\frac{d s_{r}}{d t} & =-\frac{1}{Y} \mu\left(s_{r}\right) x_{r}+\frac{Q_{1}}{V_{r}}\left(s_{1}-s_{r}\right) \\
\frac{d x_{r}}{d t} & =\mu\left(s_{r}\right) x_{r}-\frac{Q_{1}}{V_{r}} x_{r}
\end{aligned}
$$

where $\mu$ is the specific growth rate of the microorganisms. As usual, we assume the yield coefficient $Y$ equal to 1 (at the price of changing the $x_{r}$ unit).

Assumption $1 \mu(\cdot)$ is a continuously differentiable, increasing and concave function on $\mathbb{R}_{+}$with $\mu(0)=0$.

The target to be reached in minimal time is defined by a threshold $\underline{s}>0$ on both zones:

$$
\mathcal{T}:=\left\{s \in \mathbb{R}_{+}^{2} \mid \max \left\{s_{1}, s_{2}\right\} \leq \underline{s}\right\}
$$

with the controls $\left(Q_{1}, Q_{2}\right) \in\left[0, \bar{Q}_{1}\right] \times\left[0, \bar{Q}_{2}\right]$.

Since the volumes $V_{1}$ and $V_{2}$ are assume to be very large compared to $V_{r}$, we face a slow-fast dynamic in which the slow variables are $\left(s_{1}, s_{2}\right)$ and the fast one are $\left(x_{r}, s_{r}\right)$. We posit $\alpha_{i}=V / V_{i}, \bar{D}_{i}=\bar{Q}_{i} / V_{r}$ for $i=1,2$ and $\epsilon=$ $V_{r} / V$ so that the system (1)-(2) can be written as

$$
\begin{aligned}
\frac{d s_{1}}{d t} & =\epsilon \alpha_{1}\left(u_{1} \bar{D}_{1}+u_{2} \bar{D}_{2}\right)\left(s_{2}-s_{1}\right) \\
\frac{d s_{2}}{d t} & =\epsilon \alpha_{2}\left[u_{1} \bar{D}_{1}\left(s_{r}-s_{2}\right)+u_{2} \bar{D}_{2}\left(s_{1}-s_{2}\right)\right] \\
\frac{d s_{r}}{d t} & =-\mu\left(s_{r}\right) x_{r}+u_{1} \bar{D}_{1}\left(s_{1}-s_{r}\right) \\
\frac{d x_{r}}{d t} & =\mu\left(s_{r}\right) x_{r}-u_{1} \bar{D}_{1} x_{r}
\end{aligned}
$$

where $u_{i}=Q_{i} / \bar{Q}_{i}(i=1,2)$ are controls in $[0,1]$. Applying Tikhonov's Theorem (see for instance) [6] in the 
time scale $\tau=\epsilon t$ the $\left(s_{r}, x_{r}\right)$ variables can be approximated by the quasi-stationary state

$$
\begin{aligned}
& s_{r}^{q s}\left(s_{1}, u_{1}\right)=\sup _{s<s_{1}}\left\{s \mid \mu(s)<u_{1} \bar{D}_{1}\right\} \\
& x_{r}^{q s}\left(s_{1}, u_{1}\right)=s_{1}-s_{r}^{q s}\left(s_{1}, u_{1}\right)
\end{aligned}
$$

which is uniquely defined and globally asymptotically stable equilibrium when $s_{1}$ and $u_{1}$ are constant, under Assumption 1. Remark that for $u_{1} \geq \mu\left(s_{1}\right) / \bar{D}_{1}$, one has $s_{r}^{q s}=s_{1}$, which corresponds to the washout of the reactor $\left(x_{r}^{q s}=0\right)$ or equivalently to a by-pass of the bioreactor, and the first pump acts as a recirculation pump. Then, the dynamics of the slow variables $\left(s_{1}, s_{2}\right)$ can be approached using the reduced dynamics

$$
\begin{aligned}
& \dot{s}_{1}=\frac{d s_{1}}{d \tau}=\alpha_{1}\left(u_{1} \bar{D}_{1}+u_{2} \bar{D}_{2}\right)\left(s_{2}-s_{1}\right) \\
& \dot{s}_{2}=\frac{d s_{2}}{d \tau}=\alpha_{2}\left[u_{1} \bar{D}_{1}\left(s_{r}^{q s}\left(s_{1}, u_{1}\right)-s_{2}\right)+u_{2} \bar{D}_{2}\left(s_{1}-s_{2}\right)\right]
\end{aligned}
$$

In the following, we shall denote by $s$ the vector in dimension 2. From now, we work with dynamics (6) with measurable controls $u=\left(u_{1}, u_{2}\right)$ in $U:=[0,1]^{2}$. Notice that the dynamics is non-linear w.r.t. control $u_{1}$ and linear w.r.t. $u_{2}$. As we shall see later in the analysis of the minimal time problem, the consideration of a second control $u_{2}$ changes significantly the optimal control problem, compared to the former works $[3,9])$. Let consider the maximal steady-state concentration at the output of bioreactor

$$
\bar{s}:=\sup \left\{s \mid \mu(s)<\bar{D}_{1}\right\}
$$

Notice that having $s_{1}<\bar{s}$ implies that the maximal flow rate $\bar{Q}_{1}$ is large enough to allow $s_{r}^{q s}$ to take any value in $\left[0, s_{1}\right]$.

Lemma 2 The domains $\mathcal{D}=\left\{s \in \mathbb{R}_{+}^{2} \mid s_{2} \leq s_{1}<\bar{s}\right\}$, and $\mathcal{D}^{*}=\left\{s \in \mathcal{D} \mid s_{1}>s_{2}\right\}$ are positively invariant for any control in $U$.

PROOF. $s_{r}^{q s}$ being non decreasing w.r.t. to $s_{1}$, we deduce that the dynamics (6) is cooperative and thus leaves the positive cone invariant [11]. Then, the only possible way to leave the set $\mathcal{C}=\left\{s \in \mathbb{R}_{+}^{2} \mid s_{1} \geq s_{2}\right\}$ is through $s_{1}=s_{2}$. One has

$$
\begin{aligned}
\dot{s}_{1}-\dot{s}_{2}=- & \left(s_{1}-s_{2}\right)\left(\alpha_{1} u_{1} \bar{D}_{1}+\alpha_{1} u_{2} \bar{D}_{2}+\alpha_{2} u_{2} \bar{D}_{2}\right) \\
& -\alpha_{2} u_{1} \bar{D}_{1}\left(s_{r}^{q s}\left(s_{1}, u_{1}\right)-s_{1}\right)
\end{aligned}
$$

At $s_{1}=s_{2}$, one obtains $\dot{s}_{1}-\dot{s}_{2} \geq 0$ whatever is the control, and we conclude that the set $\mathcal{C}$ is invariant. From equations (6), one has $\dot{s}_{1} \leq 0$ in $\mathcal{C}$. Therefore, $s_{1}$ is non increasing with time and the set $\mathcal{D}$ is then invariant. Moreover one has

$$
\dot{s}_{1}-\dot{s}_{2} \geq-\left(s_{1}-s_{2}\right)\left(\alpha_{1} \bar{D}_{1}+\alpha_{1} \bar{D}_{2}+\alpha_{2} \bar{D}_{2}\right)
$$

from which we deduce, by Gronwall's Lemma that $s_{1}-$ $s_{2}=0$ cannot be reached in finite time, which proves the invariance of the set $\mathcal{D}^{*}$.

From now on, we consider initial conditions in $\mathcal{D}$ (typically $s_{1}(0)=s_{2}(0)$ for a uniform pollution). The target can then be simply written as $\mathcal{T}=\left\{s \in \mathcal{D} \mid s_{1} \leq \underline{s}\right\}$.

Lemma 3 From any initial condition in $\mathcal{D} \backslash \mathcal{T}$, the target can be reached in finite time.

PROOF. In the domain $\mathcal{D}$, the feedback control $u_{1}\left(s_{1}\right)$ defined such that $s_{r}^{q s}\left(s_{1}, u_{1}\left(s_{1}\right)\right)=s_{1} / 2$ is admissible, and with the control $u_{2}=0$, the dynamic of the variable $m=s_{1} / \alpha_{1}+s_{2} / \alpha_{2}$ is $\dot{m}=-\mu\left(s_{1} / 2\right) s_{1} / 2$. Posit $k=$ $1 / \alpha_{1}+1 / \alpha_{2}$ and one has $m \leq k s_{1}$. As $\mu$ is increasing, one obtains $\dot{m} \leq-\mu(m /(2 k)) m /(2 k)$ which shows that $m$ converges asymptotically to 0 , and thus $s$ as well. The target is then reached in finite time.

\section{Study of the minimal time problem}

Let us write the Hamiltonian, where $\lambda=\left(\lambda_{1}, \lambda_{2}\right)$

$$
\begin{aligned}
H(s, \lambda, u)= & u_{1} \bar{D}_{1}\left[\alpha_{1} \lambda_{1}\left(s_{2}-s_{1}\right)+\alpha_{2} \lambda_{2}\left(s_{r}^{q s}-s_{2}\right)\right] \\
& +u_{2} \bar{D}_{2}\left(\alpha_{2} \lambda_{2}-\alpha_{1} \lambda_{1}\right)\left(s_{1}-s_{2}\right)-\lambda_{0}
\end{aligned}
$$

(where $\lambda_{0}$ equals 0 or 1 ) which satisfies $\max _{u} H(s, \lambda, u)=$ 0 along any extremal. The transversality conditions are

$$
\lambda_{1}\left(t_{f}\right)<0, \quad \lambda_{2}\left(t_{f}\right)=0
$$

where $t_{f}$ denotes the first entry time in the target. Therefore abnormal extremal with $\lambda_{0}=0$ does not exist as it would imply $\lambda \equiv 0$. Notice that $s_{r}^{q s}$ is non-differentiable exactly at $\left(s_{1}, u_{1}\right)$ with $u_{1} \bar{D}_{1}=\mu\left(s_{1}\right)$. If $u_{1}^{\star}$ fulfills this case, one has $\left.u_{1}^{\star} \in\right] 0,1\left[\left(\right.\right.$ as $\left.s_{1}<\bar{s}\right)$ and $H=\left(u_{1} \bar{D}_{1}+\right.$ $\left.u_{2} \bar{D}_{2}\right)\left(\alpha_{2} \lambda_{2}-\alpha_{1} \lambda_{1}\right)\left(s_{1}-s_{2}\right)-1$ for any $u_{1}>u_{1}^{\star}$. Then $\left(\alpha_{2} \lambda_{2}-\alpha_{1} \lambda_{1}\right)\left(s_{1}-s_{2}\right)$ has to be non-positive, which contradicts $H=0$ along any extremal of the (non-smooth) Maximum Principle [12]. Therefore, we can consider the smooth Maximum Principle and $\lambda$ is solution of the adjoint system $\dot{\lambda}=-\partial_{s} H\left(s, \lambda, u^{\star}\right)$ :

$$
\begin{aligned}
& \dot{\lambda}_{1}=\alpha_{1} \lambda_{1}\left(u_{1}^{\star} \bar{D}_{1}+u_{2}^{\star} \bar{D}_{2}\right)-\alpha_{2} \lambda_{2}\left(u_{1}^{\star} \bar{D}_{1} \partial_{s_{1}} s_{r}^{q s}+u_{2}^{\star} \bar{D}_{2}\right) \\
& \dot{\lambda}_{2}=-\alpha_{1} \lambda_{1}\left(u_{1}^{\star} \bar{D}_{1}+u_{2}^{\star} \bar{D}_{2}\right)+\alpha_{2} \lambda_{2}\left(u_{1}^{\star} \bar{D}_{1}+u_{2}^{\star} \bar{D}_{2}\right)
\end{aligned}
$$

with boundary conditions (7). 
Lemma 4 Along any extremal, one has $\lambda_{1}(t)<0$ for any $t \leq t_{f}$, and $\lambda_{2}(t)<0$ for any $t<t_{f}$.

PROOF. At states such that $s_{r}^{q s}\left(s_{1}, u_{1}^{\star}\right)<s_{1}$, one has $\partial_{s_{1}} s_{r}^{q s}=0$ and when $s_{r}^{q s}\left(s_{1}, u_{1}^{\star}\right)=s_{1}$, one has $\partial_{s_{1}} s_{r}^{q s}=1$. In both case, the off-diagonal terms of the Jacobian matrix of the $\lambda$ dynamics are non-positive. Therefore, the dynamics (8) is competitive or equivalently cooperative in backward time. Therefore, the positive cone is preserved by the dynamics (8) in backward time [11], which implies that one has $\lambda_{i}(t) \leq 0(i=1,2)$ for any $t \leq t_{f}$. If for some $i$ one has $\lambda_{i}(t)=0$ for $t<t_{f}$, then $\lambda_{j}(t)<0$ for $j \neq i$ (as $\lambda$ cannot be a null vector). As $H=0$ along any optimal trajectory, $u_{1}^{\star}$ and $u_{2}^{\star}$ cannot be simultaneously equal to 0 . Therefore, one has $\dot{\lambda}_{i}(t)>0$, which implies that $\lambda_{i}$ stays positive for any further time, in contradiction with the transversality conditions (7).

We can then consider the non-negative function

$$
\gamma(t)=\frac{\alpha_{2} \lambda_{2}(t)}{\alpha_{1} \lambda_{1}(t)}
$$

so that system (8) can be replaced by the single o.d.e.

$$
\begin{aligned}
\dot{\gamma}= & \left(u_{1}^{\star} \bar{D}_{1}+u_{2}^{\star} \bar{D}_{2}\right)\left[\left(\alpha_{2}-\alpha_{1}\right) \gamma-\alpha_{2}\right] \\
& +\left(u_{1}^{\star} \bar{D}_{1} \partial_{s_{1}} s_{r}^{q s}+u_{2}^{\star} \bar{D}_{2}\right) \alpha_{1} \gamma^{2}
\end{aligned}
$$

with the boundary condition

$$
\gamma\left(t_{f}\right)=0
$$

Notice that for $s_{1}=s_{2}$ the maximization of the Hamiltonian gives $\left.u_{1}^{\star} \in\right] 0,1\left[\right.$ as $\lambda_{2}$ is negative, which implies $\dot{s}_{1}-\dot{s}_{2}>0$. Therefore, any extremal satisfies $s(t) \in \mathcal{D}^{*}$ for any $t>0$ and one can write the Hamiltonian as

$$
\begin{gathered}
H=-\alpha_{1} \lambda_{1} \bar{D}_{1}\left(s_{1}-s_{2}\right) u_{1}\left[1+\gamma \frac{s_{2}-s_{r}^{q s}}{s_{1}-s_{2}}\right] \\
-\alpha_{1} \lambda_{1} \bar{D}_{2}\left(s_{1}-s_{2}\right) u_{2}[1-\gamma]-1
\end{gathered}
$$

where $u_{1}$ has to maximize the non-linear function

$$
u_{1} \mapsto \phi\left(s, \gamma, u_{1}\right):=u_{1}\left[1-\gamma \frac{s_{r}^{q s}\left(s_{1}, u_{1}\right)-s_{2}}{s_{1}-s_{2}}\right]
$$

which is non differentiable at $\tilde{u}_{1}:=\mu\left(s_{1}\right) / \bar{D}_{1}$ but one has

$$
\begin{aligned}
\psi(s, \gamma) & :=\lim _{u_{1} \rightarrow \tilde{u}_{1}, u_{1}<\tilde{u}_{1}} \partial_{u_{1}} \phi\left(s, \gamma, \tilde{u}_{1}\right) \\
& =(1-\gamma)-\frac{\gamma \mu\left(s_{1}\right)}{\mu^{\prime}\left(s_{1}\right)\left(s_{1}-s_{2}\right)}
\end{aligned}
$$

We define $\hat{u}_{1}=\hat{u}_{1}(s, \gamma):=\operatorname{argmax}_{u_{1} \in\left[0, \tilde{u}_{1}\right]} \phi\left(s, \gamma, u_{1}\right)$.
Lemma 5 Under Assumption 1, for $s_{1}>s_{2}>0$ and $\gamma>0$, the maximization of $H$ w.r.t. $u_{1}$ is achieved - at $\left.u_{1}=\hat{u}_{1}(s, \gamma) \in\right] 0, \tilde{u}_{1}[$ which happens to be the unique solution of $\partial_{u_{1}} \phi=0$ when $\gamma \geq 1$ or when $\gamma<1$, $\psi(s, \gamma)<0$ and $\phi\left(s, \gamma, \hat{u}_{1}\right) \geq 1-\gamma$,

- at $u_{1}=1$ in any other case.

Moreover the maximizer is unique except when $\phi\left(s, \gamma, \hat{u}_{1}\right)=$ $1-\gamma$ where the maximizers are $\hat{u}_{1}$ and 1 .

PROOF. On $\left[0, \tilde{u}_{1}\right]$, the map $u_{1} \mapsto s_{r}^{q s}$ is increasing and convex under Assumption 1. This implies that the function $\phi$ is strictly concave w.r.t. $u_{1} \in\left[0, \tilde{u}_{1}\right]$. As one has $\phi(s, \gamma, 0)>0, \partial_{u_{1}} \phi(s, \gamma, 0)>0$ and $\phi\left(s, \gamma, \tilde{u}_{1}\right)=$ $\tilde{u}_{1}(1-\gamma)$, we deduce that the maximum of $\phi$ w.r.t. $u_{1} \in$ $\left[0, \tilde{u}_{1}\right]$ is achieved at a unique $\left.\hat{u}_{1} \in\right] 0, \tilde{u}_{1}\left[\right.$ with $\partial_{u_{1}} \phi=$ 0 exactly when $\gamma \geq 1$ or $\psi(s, \gamma)<0$. This gives the conclusion, as one has $\phi\left(s, \gamma, u_{1}\right)=u_{1}(1-\gamma)$ for $u_{1} \in$ $\left[\tilde{u}_{1}, 1\right]$.

Fig. 2 depicts the possible shapes of $\phi$ when $\gamma<1$. Notice that when it is optimal to by-pass the bioreactor, then it is optimal to use the maximal flow rate for $Q_{1}\left(u_{1}=1\right)$.

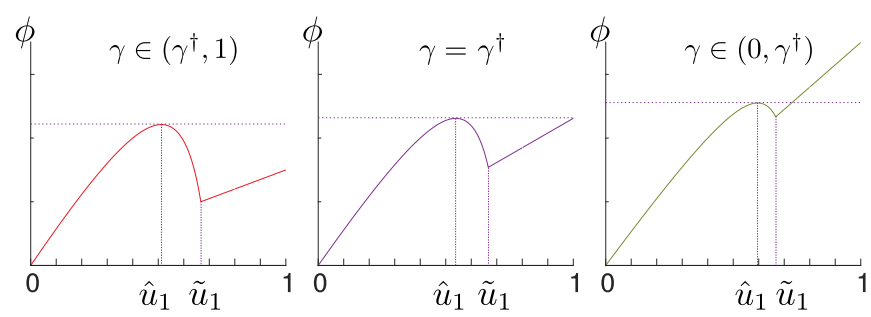

Figure 2. Three kinds of picture when maximizing the function $\phi$ (with $\gamma<1$ ), where $\gamma^{\dagger}$ is such that $\phi\left(s, \gamma^{\dagger}, \tilde{u}_{1}\right)=1-\gamma^{\dagger}$. In the middle, lack of unique maximizer.

The velocity set takes the form $F\left(s, u_{1}\right) u_{1}+G(s) u_{2}$, with

$$
\begin{aligned}
& F\left(s, u_{1}\right):=\bar{D}_{1}\left[\begin{array}{c}
\alpha_{1}\left(s_{2}-s_{1}\right) \\
\alpha_{2}\left(s_{r}^{q s}\left(s_{1}, u_{1}\right)-s_{2}\right)
\end{array}\right] \\
& G(s):=\bar{D}_{2}\left[\begin{array}{c}
\alpha_{1}\left(s_{2}-s_{1}\right) \\
\alpha_{1}\left(s_{1}-s_{2}\right)
\end{array}\right]
\end{aligned}
$$

which is non convex because $u_{1}$ does not enter linearly in the dynamics. Therefore, one cannot guarantee the existence of an optimal trajectory with the usual Filippov's Theorem. Nevertheless, it exists among relaxed controls, which amounts to consider controls $\left(u_{1}^{a}, u_{1}^{b}, p, u_{2}\right)$ in $U^{2}$ with the dynamics $\dot{s}=p F\left(s, u_{1}^{a}\right)+(1-p) F\left(s, u_{1}^{b}\right)+$ $G(s) u_{2}$ as we did in [9].

Lemma 6 An optimal solution among relaxed controls is realized with a non-relaxed control. Moreover, the only 
possibility to maintain the two maxima of $\phi$ along an extremal trajectory is with $u_{1}=1$.

PROOF. When the maximization of $H$ w.r.t. to $u_{1}$ is unique, the maximization of the relaxed Hamiltonian is reached for any $p \in] 0,1\left[\right.$ with $u_{1}^{a}=u_{1}^{b}$ or $p=1$ or $p=0$, that is for non-relaxed controls. Accordingly to Lemma 5 , the non-uniqueness of $u_{1}^{\star}$ occurs exactly when $\gamma<1$ and $\phi\left(s, \gamma, \hat{u}_{1}\right)=1-\gamma$. At such points, the relaxed controls that maximize $H$ are $\left(\hat{u}_{1}, 1, p, 1\right)$ with $p \in[0,1]$, and one has $H=\left(s_{1}-s_{2}\right)\left(\alpha_{2} \lambda_{2}-\alpha_{1} \lambda_{1}\right)\left(\bar{D}_{1}+\right.$ $\left.\bar{D}_{2}\right)-1$. Therefore, a necessary condition for having the non-uniqueness of $u_{1}^{\star}$ on a open time interval is to have $\Gamma=\left(s_{1}-s_{2}\right)\left(\alpha_{2} \lambda_{2}-\alpha_{1} \lambda_{1}\right)$ constant, with

$$
\begin{aligned}
& \dot{s}_{1}=\alpha_{1}\left[\left(p \hat{u}_{1}+1-p\right) \bar{D}_{1}+\bar{D}_{2}\right]\left(s_{2}-s_{1}\right) \\
& \dot{s}_{2}=\alpha_{2} p \hat{u}_{1} \bar{D}_{1}\left(\hat{s}_{r}^{q s}-s_{2}\right)+\alpha_{2}\left[(1-p) \bar{D}_{1}+\bar{D}_{2}\right]\left(s_{1}-s_{2}\right) \\
& \dot{\lambda}_{1}=\alpha_{1} \lambda_{1} p \hat{u}_{1} \bar{D}_{1}+\left(\alpha_{1} \lambda_{1}-\alpha_{2} \lambda_{2}\right)\left[(1-p) \bar{D}_{1}+\bar{D}_{2}\right] \\
& \dot{\lambda}_{2}=\left(\alpha_{2} \lambda_{2}-\alpha_{1} \lambda_{1}\right)\left[\left(p \hat{u}_{1}+1-p\right) \bar{D}_{1}+\bar{D}_{2}\right]
\end{aligned}
$$

where we posit $\hat{s}_{r}^{q s}=s_{r}^{q s}\left(s_{1}, \hat{u}_{1}\right)$. Calculation (given in Appendix 1) gives the expression

$$
\dot{\Gamma}=-\alpha_{2} p \hat{u}_{1} \bar{D}_{1}\left[\left(\alpha_{2} \lambda_{2}-\alpha_{1} \lambda_{1}\right)\left(\hat{s}_{r}^{q s}-s_{1}\right)+\alpha_{1} \lambda_{2}\left(s_{1}-s_{2}\right)\right]
$$

which is null only for $p=0$, that is for the non-relaxed control $u_{1}=1$.

We now present the main result of the article, that characterizes the behavior of the optimal control. Let us define the set

$$
E:=\left\{(s, \gamma) \in \mathbb{R}_{+}^{3}: \phi\left(s, \gamma, \hat{u}_{1}(s, \gamma)\right) \geq 1-\gamma\right\}
$$

whose boundary defines the locus of possible switch of $u_{1}$ between $\hat{u}_{1}$ and 1 , according to Lemma 5 .

Proposition 7 Let $s^{\star}(\cdot)$ be an optimal trajectory associated to a control $u^{\star}(\cdot)$, and $\gamma^{\star}(\cdot)$ be the solution of (10)(11). Then, there exist $0 \leq t_{1} \leq t_{2}<t_{f}$ such that for almost any $t \in\left[0, t_{f}\right]$, one has

(1) $u_{2}^{\star}(t)=0$ and $u_{1}^{\star}(t)=\hat{u}_{1}\left(s^{\star}(t), \gamma^{\star}(t)\right)$ with $t \in$ $\left[0, t_{1}\right]$, and $t_{1}$ is the first time such that $\gamma^{\star}(t) \leq 1$;

(2) $u_{2}^{\star}(t)=1$ and $u_{1}^{\star}(t)=\hat{u}_{1}\left(s^{\star}(t), \gamma^{\star}(t)\right)$ with $t \in$ $\left[t_{1}, t_{2}\right]$, and $t_{2}$ is the infimum of the times such that $\left(s^{\star}(t), \gamma^{\star}(t)\right) \notin E$

(3) $u_{2}^{\star}(t)=1$ and $u_{1}^{\star}(t)=1$ with $t \in\left[t_{2}, t_{f}\right]$.

PROOF. According to Pontryagin's Maximum Principle [8], an optimal control $u^{\star}(\cdot)$ maximizes for a.e. $t \in\left[0, t_{f}\right]$ the Hamiltonian $H$ given in (12) along an optimal pair $s^{\star}(\cdot), \lambda^{\star}(\cdot)$. Since for any $\left.t \in\right] 0, t_{f}[$ one has $\lambda_{1}^{\star}(t)>0$ and $\gamma^{\star}(t)<0$ (Lemma 4$), u_{1}^{\star}(t)$ and $u_{2}^{\star}(t)$ maximize respectively $u_{1} \mapsto \phi\left(s^{\star}(t), \gamma^{\star}(t), u_{1}\right)$ and $u_{2} \mapsto\left(1-\gamma^{\star}(t)\right) u_{2}$ over $[0,1]$. Suppose $\gamma^{\star}(0)>1$ (otherwise $\left.t_{1}=0\right)$. Since $\gamma^{\star}$ is continuous with $\gamma^{\star}\left(t_{f}\right)=0$, there exists $t_{1}=\min \left\{t \geq 0 \mid \gamma^{\star}(t)=1\right\}$. At any $t \geq$ $t_{1}$ with $\gamma^{\star}(t)=1$, one has $\dot{\gamma}^{\star}(t)=-\alpha_{1} \bar{D}_{1} u_{1}^{\star}(t)<0$ $\left(u_{1}^{\star}(t) \in\right] 0, \tilde{u}\left(s^{\star}(t)\right)[\subseteq] 0,1[$ from Lemma 5), showing that once the set $\{\gamma \leq 1\}$ is reached, it is never left in future time. Consequently, $H$ is maximized by $u_{2}^{\star}(t)=1$ at any $t>t_{1}$.

Now, Lemma 5 states that $\left(s^{\star}\left(t_{1}\right), \gamma^{\star}\left(t_{1}\right)\right) \in \operatorname{int}(E)$. On the other hand one has $\phi\left(s^{\star}\left(t_{f}\right), \gamma^{\star}\left(t_{f}\right), u_{1}\right)=u_{1}$, that is $\left(s^{\star}, \gamma^{\star}\right)\left(t_{f}\right) \notin E$. Then, there exists $t_{2}=\inf \{t \geq$ $\left.t_{1} \mid\left(s^{\star}(t), \gamma^{\star}(t)\right) \notin E\right\}<t_{f}$ with $\phi\left(s^{\star}, \gamma^{\star}, \hat{u}\left(s^{\star}, \gamma^{\star}\right)\right)=$ $1-\gamma^{\star}$ at $t_{2}$. As $u_{1}=1$ is optimal outside $E$ (Lemma $5)$, and is the only control for an extremal to stay on the boundary of $E$ (Lemma 6 ), let us consider the dynamics with $u_{1}=u_{2}=1$ :

$$
\begin{aligned}
& \dot{s}_{1}=-\alpha_{1}\left(\bar{D}_{1}+\bar{D}_{2}\right)\left(s_{1}-s_{2}\right) \\
& \dot{s}_{2}=\alpha_{2}\left(\bar{D}_{1}+\bar{D}_{2}\right)\left(s_{1}-s_{2}\right) \\
& \dot{\gamma}=-\left(\alpha_{1} \gamma+\alpha_{2}\right)\left(\bar{D}_{1}+\bar{D}_{2}\right)(1-\gamma)
\end{aligned}
$$

and the function $\varphi(t):=\phi\left(s(t), \gamma(t), \hat{u}_{1}(s(t), \gamma(t))\right)$ along its solutions. Calculation (given in Appendix 2) gives

$$
\begin{aligned}
\varphi= & 1-\gamma \Rightarrow \\
& \frac{\gamma(\dot{\varphi}+\dot{\gamma})}{\bar{D}_{1}+\bar{D}_{2}}=\hat{u}_{1}\left[\alpha_{2}(1-\gamma)^{2}-\alpha_{1} \gamma^{2}\right]-\alpha_{2}(1-\gamma)^{2}
\end{aligned}
$$

One can easily check that this last quantity is always negative, as $\hat{u}_{1}(s, \gamma)$ belongs to $] 0,1\left[\right.$. As $\gamma^{\star}(t)>0$ for any $t<t_{f}$ (Lemma 4), we conclude that the optimal trajectory has to leave the set $E$ at $t_{2}$ and cannot reach it in future time, which proves that $u_{1}^{\star}(t)=1$ is optimal any $t>t_{2}$.

Remark 8 Proposition 7 states that it is optimal to end the process with no decontamination (or bypass of the reactor), which has the effect to recontaminate the second patch (but keeping its concentration below the threshold $\underline{s})$. Moreover, $u_{2}^{\star}$ takes the value 1 when $u_{1}^{\star}$ is below 1 . This means that when it is optimal to switch on the recirculation pump, the bioreactor is not yet bypassed.

\section{Numerical simulations}

We consider the Monod growth function [7], widely used in bio-processes, which satisfies Assumption 1

$$
\mu(s)=\mu_{\max } \frac{s}{K_{s}+s}
$$

with parameters $\mu_{\max }=1\left[h^{-1}\right], K_{s}=1\left[g^{-1}\right]$, and volumes $V_{1}=300[l], V_{2}=700[l], V_{r}=2[l]$. The threshold 
level is set to $\underline{s}=1\left[g^{-1}\right]$. We first compare in Table 1 the treatment time of the strategy presented in this work (that is with the constraint $s_{r}^{q s} \in\left[0, s_{1}\right]$ ) but without recirculation, with the former strategy presented in [3] (that is with the constraint $s_{r}^{q s} \in\left[0, s_{2}\right]$ and no recirculation). We see that allowing the second zone to be recontaminated is beneficial over the treatment time, and this effect is even larger when the initial concentration in the second zone is small compared to the threshold. This effect can be attributed to the fact that under the constraint $s_{r}^{q s} \in\left[0, s_{2}\right]$ the variable $s_{2}$ can only be decreasing, providing a much more restricted control set than the constraint $s_{r}^{q s} \in\left[0, s_{1}\right]$, especially for initial conditions $s_{2}(0)$ close to 0 . When the initial concentration is better mixed, this effect is less significant.

In Figure 3 we illustrate the impact of the maximum recirculation rate on the treatment time with the optimal strategy given in Proposition 7. We give the gains compared with no recirculation. One can see that increasing the maximal recirculation rate is beneficial, and as before, the results are even better when the inhomogeneity is initially large.

\begin{tabular}{||c||c|c|c||}
\hline \hline $\begin{array}{c}\text { Initial } \\
\text { condition }\end{array}$ & $\begin{array}{c}\text { Optimal time } \\
\text { in [3] }\end{array}$ & $\begin{array}{c}T_{\text {opt }} \\
\bar{Q}_{1}=2.5\end{array}$ & $\begin{array}{c}T_{\text {opt }} \\
\bar{Q}_{1}=10\end{array}$ \\
\hline \hline$(2.5,0.1)$ & 1593.6 & 187.9 & 46.8 \\
Gain & & $88.2 \%$ & $97.1 \%$ \\
$(2.5,0.5)$ & 624.0 & 351.7 & 175.3 \\
Gain & & $43.6 \%$ & $71.9 \%$ \\
$(2.5,1.5)$ & 1293.6 & 1173.6 & 1039.2 \\
Gain & & $9.3 \%$ & $19.7 \%$ \\
$(2.5,2.5)$ & 1916.6 & 1806.5 & 1676.1 \\
Gain & & $5.8 \%$ & $12.6 \%$ \\
\hline \hline
\end{tabular}

Table 1. Comparison of optimal treatment times, where $T_{\text {opt }}$ is the minimal time with recirculation.

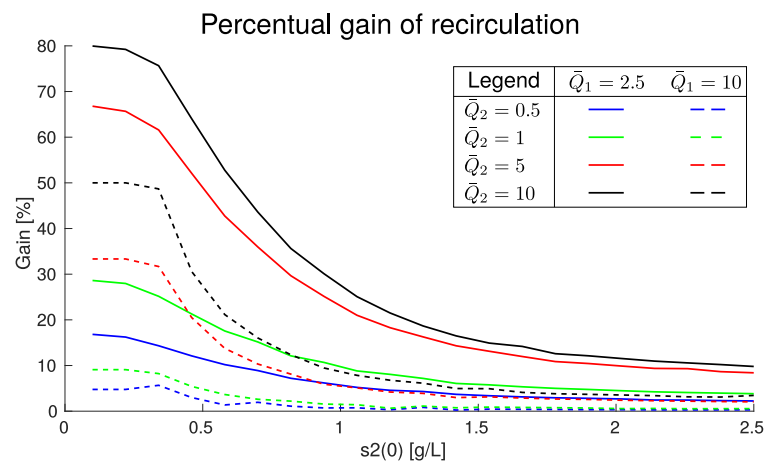

Figure 3. Gain of maximum recirculation speed compared to no recirculation.
Several optimal trajectories in the phase portrait (obtained by backward integration) are drawn in Fig. 4-6. The blue parts represent no saturation of both (optimal) controls (point 1. of Proposition 7). The green ones correspond to optimal $Q_{2}$ taking its maximum value (point 2 . of Proposition 7). In red, optimal $Q_{2}$ takes its maximum value while optimal $s_{r}^{\text {qs }}$ is equal to $s_{1}$, which amounts to bypass the bioreactor (point 3. of Proposition 7).

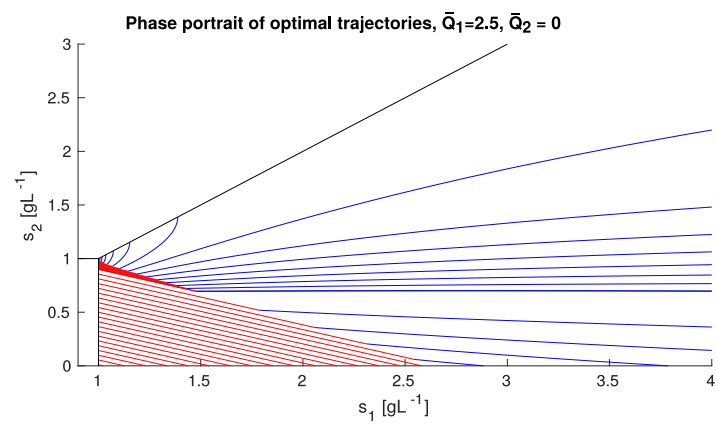

Figure 4. Extremals field with $\bar{Q}_{2}=0$.

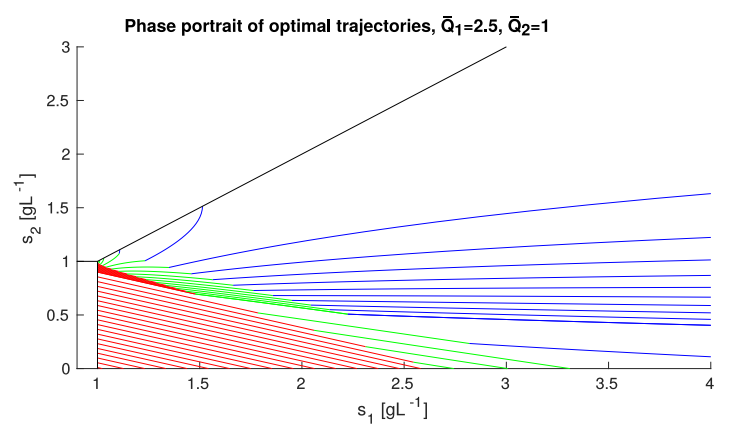

Figure 5. Extremals field with $\bar{Q}_{2}=1$.

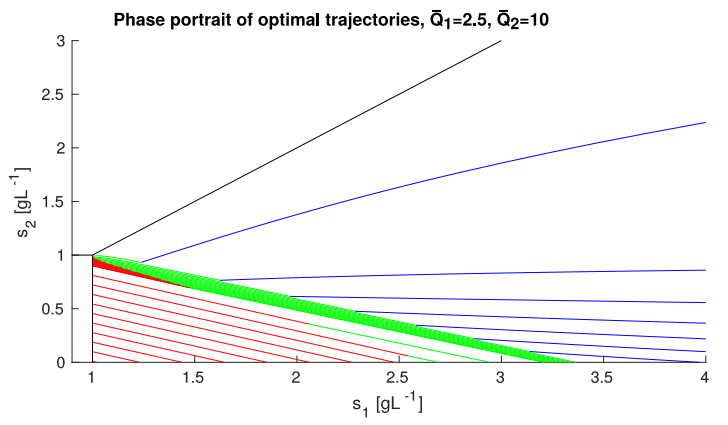

Figure 6. Extremals field with $\bar{Q}_{2}=10$.

\section{Conclusions}

In this work we have shown the benefit of mixing water of a contaminated resource to be treated, but at some precise time and duration to be determined optimally. Moreover, we have shown the non-intuitive fact that a recontamination of the area where treated water is released 
is beneficial when operated with a maximal mixing before the end of treatment (this last stage consisting in a by-pass of the bioreactor). Indeed, we have shown that the optimal control performs an over-decontamination in the released area, that is followed by the fastest homogenization of the concentrations between the two areas.

The switching times between the three stages of the optimal strategy rely on the knowledge of one adjoint variable, and therefore cannot be given as a simple feedback as it was the case in the former work [3]. However, with a proper estimation of the parameters of the model it is not difficult to perform backward integration to determine the adjoint function and then the switching times. Measurements of the initial concentrations at two remote locations in the resource, and of the initial speed of variation of these concentrations are enough for the identification of the inhomogeneity parameter of the model, and to determine the optimal duration. Then, the application of the optimal policy requires the on-line measurement of the pollutant concentration at the same two locations of the resource.

Finally, the methodology we have deployed here considering a new additional control can be applied to other problems with inhomogeneous liquid media for which recirculation or mixing can be handled, and then considered as an additional control variable.

\section{Acknowledgements}

This work was developed in the context of the 'DYMECOS' INRIA Associated team and the project BIONATURE of CIRIC INRIA CHILE. It was also partially supported by CONICYT grants REDES 130067 and 150011. The second author was supported by FONDECYT projects 1110888 and 1160204, BASAL project (Centro de Modelamiento Matemático, Universidad de Chile), MathAmsud N¹5MATH-02, and CONICYT-PCHA Doctorado Nacional 2013 folio 21130840 .

The authors are grateful to Prof. P. Gajardo and A. Rousseau for fruitful discussions and insightful ideas.

\section{References}

[1] S. Barbier, A. Rapaport, and A. Rousseau. Modelling of biological decontamination of a water resource in natural environment and related feedback strategies. Journal of Scientific Computing, pages 1-14, 2016.

[2] P. Gajardo, J. Harmand, H. Ramirez, A. Rapaport, V. Riquelme, and A. Rousseau. Modeling and control of in-situ decontamination of large water resources. ESAIM: ProcS, 57:70-85, 2017.

[3] P. Gajardo, J. Harmand, H. Ramírez C., and A. Rapaport. Minimal time bioremediation of natural water resources. Automatica J. IFAC, 47(8):1764-1769, 2011.
[4] P. Gajardo, H. Ramírez, V. Riquelme, and A. Rapaport. Bioremediation of natural water resources via optimal control techniques. In BIOMAT 2011, pages 178-190. World Sci. Publ., Hackensack, NJ, 2012.

[5] J. Harmand, C. Lobry, A. Rapaport, and T. Sari. The Chemostat: Mathematical Theory of Microorganims Cultures, volume 1 of Chemical Engineering Series, Chemostat and Bioprocesses Set,. ISTE-Wiley, 2017.

[6] H.K. Khalil. Nonlinear Systems. Pearson Education. Prentice Hall, 2002.

[7] J. Monod. La technique de la culture continue, théorie et applications. Annales de l'Institut Pasteur, 79(4):390-410, 1950.

[8] L. S. Pontryagin, V. G. Boltyanskii, R. V. Gamkrelidze, and E. F. Mishechenko. The Mathematical Theory of Optimal Processes. John Wiley \& Sons, Newy-York/London, 1962.

[9] H. Ramírez, A. Rapaport, and V. Riquelme. Optimal Feedback Synthesis and Minimal Time Function for the Bioremediation of Water Resources with Two Patches. SIAM J. Control Optim., 54(3):1697-1718, 2016.

[10] E. Rurangwa and M.C.J. Verdegem. Microorganisms in recirculating aquaculture systems and their management. Reviews in aquaculture, 7(2):117-130, 2015.

[11] H.L. Smith. Monotone dynamical systems, volume 41 of Mathematical Surveys and Monographs. American Mathematical Society, Providence, RI, 1995. An introduction to the theory of competitive and cooperative systems.

[12] R. Vinter. Optimal control. Systems \& Control: Foundations \& Applications. Birkhäuser Boston, 2000.

\section{Appendix 1}

Posit $\tilde{s}=s_{1}-s_{2}, \tilde{\lambda}=\alpha_{2} \lambda_{2}-\alpha_{1} \lambda_{1}$ and $\tilde{D}=(1-p) \bar{D}_{1}+$ $\bar{D}_{2}$. One has

$$
\begin{aligned}
& \dot{\tilde{s}}=-\alpha_{1}\left(p \hat{u}_{1} \bar{D}_{1}+\tilde{D}\right) \tilde{s}-\alpha_{2} p \hat{u}_{1} \bar{D}_{1}\left(\hat{s}_{r}^{q s}-s_{2}\right)-\alpha_{2} \tilde{D} \tilde{s} \\
& \dot{\tilde{\lambda}}=\alpha_{2} \tilde{\lambda}\left(p \hat{u}_{1} \bar{D}_{1}+\tilde{D}\right)-\alpha_{1}^{2} \lambda_{1} p \hat{u}_{1} \bar{D}_{1}+\alpha_{1} \tilde{\lambda} \tilde{D}
\end{aligned}
$$

and then

$$
\begin{array}{r}
\dot{\Gamma}=\dot{\tilde{s}} \tilde{\lambda}+\dot{\tilde{\lambda}} \tilde{s}=-\alpha_{1} p \hat{u}_{1} \bar{D}_{1} \tilde{s} \tilde{\lambda}-\alpha_{2} p \hat{u}_{1} \bar{D}_{1}\left(\hat{s}_{r}^{q s}-s_{2}\right) \tilde{\lambda} \\
+\alpha_{2} \tilde{\lambda}_{p} \hat{u}_{1} \bar{D}_{1} \tilde{s}-\alpha_{1}^{2} \lambda_{1} p \hat{u}_{1} \bar{D}_{1} \tilde{s}
\end{array}
$$

Factorizing $\alpha_{2}$ and $\alpha_{1}$, on obtains

$$
\dot{\Gamma}=-\alpha_{2} p \hat{u}_{1} \bar{D}_{1}\left(\hat{s}_{r}^{q s}-s_{1}\right) \tilde{\lambda}-\alpha_{1} p \hat{u}_{1} \bar{D}_{1} \tilde{s}\left(\tilde{\lambda}+\alpha_{1} \lambda_{1}\right)
$$

or equivalently

$\dot{\Gamma}=-\alpha_{2} p \hat{u}_{1} \bar{D}_{1}\left[\left(\alpha_{2} \lambda_{2}-\alpha_{1} \lambda_{1}\right)\left(\hat{s}_{r}^{q s}-s_{1}\right)+\alpha_{1} \lambda_{2}\left(s_{1}-s_{2}\right)\right]$

\section{Appendix 2}

As $\hat{u}_{1}$ is a maximizer of $\phi$ on the interior of $\left[0, \tilde{u}_{1}\right]$, the time derivative of $\varphi$ is simply

$$
\dot{\varphi}=\hat{u}_{1}\left[\gamma \frac{\dot{s}_{2}}{s_{1}-s_{2}}+\left(\gamma \frac{\dot{s}_{1}-\dot{s}_{2}}{s_{1}-s_{2}}-\dot{\gamma}\right) \frac{\hat{s}_{r}^{q s}-s_{2}}{s_{1}-s_{2}}\right]
$$


Then, one has from equations (14)

$$
\frac{\dot{\varphi}}{\bar{D}_{1}+\bar{D}_{2}}=\hat{u}_{1}\left[\gamma \alpha_{2}+\left(\alpha_{2}-2 \alpha_{2} \gamma-\alpha_{1} \gamma^{2}\right) \frac{\hat{s}_{r}^{q s}-s_{2}}{s_{1}-s_{2}}\right]
$$

and under the condition $\varphi=1-\gamma$ with expression (13)

$$
\begin{aligned}
\frac{\gamma \dot{\varphi}}{\bar{D}_{1}+\bar{D}_{2}}= & \hat{u}_{1}\left[\alpha_{2}(1-\gamma)^{2}-\alpha_{1} \gamma^{2}\right] \\
& +(\gamma-1)\left(\alpha_{2}-2 \alpha_{2} \gamma-\alpha_{1} \gamma^{2}\right)
\end{aligned}
$$

Finally, with the expression of $\dot{\gamma}$ from (14), on obtains

$$
\frac{\gamma(\dot{\varphi}+\dot{\gamma})}{\bar{D}_{1}+\bar{D}_{2}}=\hat{u}_{1}\left[\alpha_{2}(1-\gamma)^{2}-\alpha_{1} \gamma^{2}\right]-\alpha_{2}(1-\gamma)^{2}
$$

